\title{
Superior vena cava obstruction with thrombolytic therapy in primary antiphospholipid syndrome
}

\author{
Manal Adel ${ }^{1}$, Marwa Mashaal ${ }^{1}$, Kareem Mahmoud ${ }^{1}$, Shady Mashhour², and Haytham \\ Soliman $^{1}$ \\ ${ }^{1}$ Cardiovascular \\ ${ }^{2}$ Radiology
}

January 30, 2021

\begin{abstract}
We report a case of superior vena cava obstruction of a 39 years old female patient with primary antiphospholipid syndrome presented with upper limb pain and swelling, which improved on thrombolytic therapy, we review patients with Antiphospholipid syndrome and management with thrombolytic therapy .
\end{abstract}

\section{Case report}

Superior vena cava obstruction with thrombolytic therapy in primary antiphospholipid syndrome

Manal Adel ,Kareem Mahmoud ,Haithem soliman ,Shady Mashoor ,Marwa Mashaal Cardiovascular department ,Kasr AlAiny Hospital ,Cairo University /Egypt

Key words

SVC superior vena cava - APS antiphospholipid syndrome

\section{Abstract}

We report a case of superior vena cava obstruction of a 39 years old female patient with primary antiphospholipid syndrome presented with upper limb pain and swelling, which improved on thrombolytic therapy ,we review patients with Antiphospholipid syndrome and management with thrombolytic therapy .

\section{Background}

Superior vena cava syndrome results from the obstruction of blood flow through the superior vena cava and is most often due to thoracic malignancy. However, benign etiologies are on the rise secondary to more frequent use of intravascular devices such as central venous catheters and pacemakers, There also are other rare non neoplastic diseases that can cause SVCS, for example, fibrosing mediastinitis, ${ }^{(1.2)}$ which is a rare chronic inflammatory condition similar to retroperitoneal fibrosis, use for indwelling catheter and hypercoagulable condition as primary antiphospholipid syndrome which is very rare condition .

\section{Case description}

39 years old female presented with fever and shortness of breath for 14 days for which Total leucocytic shift 16 with shift to the left ,hemoglobin 9.5 MCV 69 ,CRP 161 , chest X ray was free on admission , ECG showed normal sinus rythm ,Echocardiography showed from right atrial mass $2.7 \times 1.9 \mathrm{~cm}$,moderate mitral and tricuspid regurgitation second day after admission she started to suffer from pain and swelling of both upper limb .Duplex Upper limbs revealed bilateral total occlusion of subclavian, axillary and brachial veins 
.Proximal part of right IJV was totally occluded and left side was partially occluded . By examination her blood pressure was 120/60, regular heart rate and rythm and oxygen saturation $97 \%$ her neck showed visible dilated non pulsating veins .cardiovascular exam showed pansystolic murmur over apex with no thrill and another at lower part of sternum increases with inspiration .lung auscultation showed diminished air entry at the right base .

During hospital stay lupus anticoagulant and anticardiolipin IgM were positive, work up against infective endocarditis was done to exclude secondary infection of right atrial mass and it was negative, tumor markers were negative , CT chest revealed right sided pleural effusion,left lower lobe consolidation ,enlarged pretracheal ,supracarinal and aortocaval lymph nodes. TEE and CMR revealed right atrial mass $2.7 \times 2.9 \times 1.7$ $\mathrm{cm}$ most likely thrombus and another mass in right atrial appendage $1.0 \times 0.7 \times 0.7 \mathrm{~cm}$, moderate mitral and tricuspid regurgitation, pulmonary hypertension ,patent non occluding thrombi within SVC.

Patient underwent venous angiography for which catheter was inserted through venous access to right cephalic vein and 1.5 million IU of streptokinase was given and then she was maintained on heparin infusion with adjustment to aPTT 50-70, venography was done and revealing no improvement so a decision was given to give second dose streptokinase on fifth day she was given second bolus 250,000 and maintained on 100,000 IU / hour for 10 hours stopped due to fever(drug reaction), patient underwent venous angiography follow up which revealed marked improvement in venous blood flow .she was discharged home safely on aspirin $75 \mathrm{mg}$ once and warfarin with proper adjustment to target INR .

\section{Outcome}

She had smooth course with improvement to her symptoms and upper limb edema and was discharged home at stable condition on regular follow up to her anticoagulation

\section{Discussion}

Benign Superior vena cava syndrome accounts for less than $10 \%$. Half of them due to fibrosing mediastinitis and use of indwelling catheters which increase incidence of thrombosis .

Antiphospholilpid syndrome is a thrombophilic syndrome in which venous or arterial thrombosis or both,may occur in patient with antiphospholipid antibodies positive , the diagnosis of definite antiphospholipid syndrome must include at least one clinical event of arterial or venous thrombosis and at least one of the laboratory criteria [lupus anticoagulant or anticardiolipin IgG or IgM antibodies, this was reported in three case at Iran Journal of Immunology ${ }^{(3)}$, Spain ${ }^{(4)}$ and Israel $^{(5)}$.

The presence of a thrombus should be considered in patients with acute onset of symptoms, occlusions that can easily be traversed by a wire, and when SVCS are secondary to central venous catheters. In these patients, a trial of systemic anticoagulation or, in the acute setting, thrombolysis can be attempted by using thrombolytic agents delivered at the site of the clot through a catheter, which allows more effective local action. Reports of systematic administration of thrombolytic agents have also been described ${ }^{(6)}$. By reducing the thrombus load, the length of the obstruction can often be reduced, thus reducing the number of stents needed. (7) However, thrombolysis is most effective if it is started within 2-5 days of onset of symptoms and tends to be ineffective if started after 10 days $^{(8)}$.

Gray et al ${ }^{(9)}$ found that $88 \%$ of the cases had a successful outcome when thrombolytic treatment was commenced within 5 days. The success rate dropped to $25 \%$ when it was started after 5 days ${ }^{(9)}$. They also observed that catheter-related SVCS demonstrated a better response to thrombolysis, which was attributed to the earlier detection of symptoms secondary to catheter dysfunction, the absence of extrinsic compression, and the ability to infuse the thrombolytic agent directly to the site of the thrombus via the existing catheter ${ }^{(11)}$. Caution should be exercised in patients with cystic fibrosis because an increased risk of hemoptysis and gastrointestinal bleeding has been reported in this patient population ${ }^{(6)}$.

Newer techniques that use pharmacomechanical thrombolysis tools, such as the Trellis Thrombectomy System (Bacchus Vascular, Santa Clara, CA) and Trellis infusion system (Covidien, Santa Clara, CA), have shown 
promising results in reducing the thrombus load ${ }^{(10,11)}$.

\section{References}

1.Rachapalli V, Boucher LM. Superior vena cava syndrome: role of the interventionalist. Canadian Association of Radiologists Journal. 2014 May 1;65(2):168-76.

2-Bays S,Rajakauna C,Sheffield $\mathrm{E}$, et al fibrosing mediastintis as a case of superior vena cava syndrome .Eur J cardiothoracic surg $2004: 26: 453-5$

3-Hegazi M, Mourou M, Hassanien O,et al.antiphospholipid syndrome presenting with superior vena cava thrombosis Iranian J of immunol 2009;6(2):103-106

4-Alcazar Ramirez J,Fernandez Nebro A,Abarca M ,et al. Central vein thrombosis in the antiphospholipid syndrome.Lupus1997;549:551-6

5-Herscovici R,Szyper KM,Ailtman A,et al.superior vena cava syndrome-changing etiology in the third millennium .lupus2012;93-96-21

6- Hassan T ,Chotirmall SH ,low TB,et al . Thrombolysis for Indwelling catheter related thrombosis and superior vena cava obstruction in cystic fibrosis;a case series Ir J Med Sci 2010;179:496-70

7-Kee ST,Kinoshita L,Razavi MK,etal.superior vena cava syndrome ; treatment with catheter directed thrombolysis and endovascular stent placement .Radiology 1998;206:187-93

8-Sriramnaveen P,Kumar VS,Kishore CK,et al.delayed presentation of superior vena cava syndrome after hemodialysis catheter removal ,Saudi JKidney Dis Transpl 2011;22:554-6

9-Gray BH,Olin JW,Graor RA,et al .Safety and efficacy of thrombolytic therapy for superior vena cava syndrome .chest 1991;99:54-9

10-Canales JF,Cardenas JC,Dougherty K, et al.single center experience with percutaneous endovascular repair of superior vena cava syndrome .catheter cardiovasc interv 2011;77:733-9

11-O'sullivan GJ,Mhuricheartaigh JN,Ferguson D,et al .Isolated pharamcomechanical thrombolysis plus primary stenting in a single procedure to treat acute thrombotic superior vena cava syndrome $\mathrm{J}$ Endovasc Ther 2010;17:115-23

\section{Hosted file}

Figures of Case.pdf available at https://authorea.com/users/392766/articles/506561-superiorvena-cava-obstruction-with-thrombolytic-therapy-in-primary-antiphospholipid-syndrome 